\title{
Testing of antibiotic combinations in NDM-1-producing nosocomial carbapenem-resistant Acinetobacter baumannii
}

\author{
Shimaa M. Shabayek ${ }^{*}$, Dalia A. El-Damasy ${ }^{2}$, Omayma M. Hassanin ${ }^{3}$ and Sahar M. Radwan' \\ ${ }^{1}$ Microbiology and Immunology Department, Faculty of pharmacy-Girls, Al-Azhar University, Cairo, Egypt. \\ ${ }^{2}$ Microbiology and Immunology Department, Faculty of Pharmacy, Egyptian Russian University. \\ ${ }^{3}$ Molecular Biology Department, Faculty of Medicine Ain Shams Research Institute (MASRI), Ain-Shams University \\ Hospitals. \\ *Correspondence: shimaa.mahmoud8570@yahoo.com
}

Article history: Received 05-12-2020 Revised 24-12-2020 Accepted 07-01-2021

\begin{abstract}
Carbapenem resistant Acinetobacter baumannii (CRAB) have become a serious problem in health care settings. Its high prevalence has been associated with nosocomial transmission, high mortality rates, drug resistance and massive economic loss. The most frequent mechanisms of resistance are carbapenemhydrolyzing class D $\beta$-lactamases (CHDL), followed by class B metallo- $\beta$-lactamases (MBL). New Delhi metallo- $\beta$-lactamase-1 (NDM-1) generated much global alarm and were labeled as superbugs which had documented impossible to be treated. Preceding our study, eight different antibiotic combinations were evaluated using checkerboard method. This was based on the results obtained from antimicrobial susceptibility testing and phenotypic detection of metallo- $\beta$-lactamase production of 27 CRAB selected from our previous work. The existence of NDM-1 gene was tested using two different methods. All antibiotic combinations showed synergistic significant results and no antagonism activity was found. The percentage of NDM-1 gene in the first detection procedure was $12 / 27(44.44 \%)$ were positive while the other negative 15 ones, 10/15 (66.67\%) poses NDM-1were found when using the second method. PCR products were then verified by DNA sequencing. The final consensus sequences were analyzed and submitted to NCBI GenBank data base, representing accession numbers: (HQ6526091), (MK682763.1), (MK682764.1), (MK682767.1), (MN251665.1), (MN251666.1) and (MN251670.1). The alignments showed similarity ranged from 94\%$100 \%$ nucleotides identity. We concluded that detection of CRAB using accurate, rapid methods and supplying hospital laboratories with molecular department for typing of pathogenic bacteria are essential. Each hospital should establish its own policies according to their antibiogram, national and international guidelines. Primary caretakers should comply with the implemented policies.
\end{abstract}

Keywords: Acinetobacter baumannii; Resistant; Checkerboard; MBL; Sequencing; NDM-1.

\section{INTRODUCTION}

Healthcare-associated infections (HAIs) are infections acquired as a result of an episode of healthcare ${ }^{1}$. It is considered a recurrent problem identified chiefly in intensive care facilities, surgical and medical wards ${ }^{2}$. The expansion of resistance of $A$. baumanii to primary antimicrobial therapies has created a deadly combination of pathogenicity and antimicrobial resistance that plagues hospitals. It is considered the World Health Organization's number one critical priority pathogen for which new therapeutics are urgently required as hospital-acquired $A$. baumannii infections will soon be untreatable ${ }^{3}$. Current treatment options for CRAB are limited and suffer from pharmacokinetic limitations, such as high toxicity and low plasma levels. As all antimicrobials could be overcomed by the bacteria, there is an urgent need for new therapies. These new therapeutic options are either antibiotic combinations or new antimicrobial drugs ${ }^{4}$. Combined therapy may even prevent antibiotic resistance emerging during the treatment ${ }^{5}$. New Delhi metallo $\beta$ lactamase-1 (NDM-1) is a novel MBL that confers resistance to all $\beta$-lactam antibiotics ${ }^{6}$. The Plasmids carrying NDM- 1 gene also carries a number of other genes conferring resistance to all aminoglycosides, macrolides and sulphamethoxazole so these isolates are resulted to be multidrug resistant. NDM-1 producers bring several additional factors which are deeply disconcerting for public health worldwide particularly that this gene can spread at an unprecedented rate ${ }^{7}$. World Health Organization (WHO) has urged all the countries to implement infection control measures to contain the spread of these bacteria. A sensitive and rapid method for detecting NDM-1 positive bacteria would be helpful in this effort ${ }^{8}$ 


\section{METHODS}

This study was carried out in Microbiology and Immunology department, Faculty of Pharmacy for Girls, Al Azhar University and Medical Ain Shams Research Institute (MASRI) Faculty of Medicine, Ain Shams University. Twenty seven isolates were selected from seventy four clinical isolates from our previous work ${ }^{9}$ depending on the results of antimicrobial sensitivity pattern and phenotypic detection of metallo$\beta$-lactamase. These isolates were collected from hospital laboratories for inpatients admitted to Urology, Surgery, pediatric, orthopedics, GIT disorders, pediatric intensive care unit and Neonatal intensive care unit departments in addition to adult Intensive care and open heart units after 48-72 hrs. of hospitalization. The study included three hospitals that were two tertiary care hospitals in Cairo and one private tertiary care hospital in Giza during the period from July 2017 till June 2018. Identification and confirmation of species, biochemical reactions, detection of CRAB using Modified Hodge test (MHT) and Imipenem EDTA combined disc test (CDT), Antimicrobial susceptibility testing and determination of Minimum inhibitory concentrations (MICs) by E-test were detailed in the previous published research ${ }^{9}$.

\subsection{Combination of antibiotics}

Antibiotic combinations were evaluated with the checkerboard method according to Mulyaningsih et al., $2010^{10}$. Two antimicrobial agents were serially diluted in two dimensional fashions to include all combinations. Fractional Inhibitory concentration values (FICA \& FICB) and FIC index were calculated for each combination of compounds and clinical isolates using the following standard equation:

$$
\begin{gathered}
\text { FIC index }=\text { FICA }+ \text { FICB }{ }^{10} \\
\text { FICA }=\frac{\text { MIC of compound } A \text { in combination }}{\text { MIC of compound A alone }}
\end{gathered}
$$

$$
F I C B=\frac{\text { MIC of compound } B \text { in combination }}{M I C \text { of compound } B \text { alone }}
$$

Synergy was defined as FIC index $p$ - value $\leq 0.5$, Indifference or no interaction was defined as FIC index p-value in the range from 1 - 4. Antagonism was defined as FIC index p- value $>4$ and the combination was considered additive if FIC index p-value was within the range 0.5-1. All isolates were tested against eight different antibiotic combinations based on the obtained results from antimicrobial susceptibility testing in addition to the commonly used treatment antibiotics. These antibiotic combinations were:- (Tigecycline + Imipenem), (Tigecycline + Meropenem), (Colistin + Impienem ), (Colistin + Meropenem), (Tigecycline + Amikacin), (Colistin + Amikacin), (Imipenem + Amikacin) and (Meropenem + Amikacin). Antibiotic stocks of $10 \mathrm{mg} / \mathrm{ml}$ concentration were prepared and diluted to MIC required using fresh Muller Hinton Broth (MHB). Bacterial inoculum was prepared from the overnight cultures of the isolates on Mullar Hinton Agar (MHA) and compared to 0.5 Mcfarland. MHB was placed into 96-well microtiter plate. Serial dilutions of antibiotics were placed into the plate to attain the concentrations of individual antibiotic in combination. Finally, wells were inoculated with $100 \mu$ l of bacteria, compared with 0.5 Mcfarland. The contents were incubated at $37^{\circ} \mathrm{C}$ for $24 \mathrm{hrs}$. and observed for visible turbidity. The MIC of the drug combinations was defined as the concentration of no visible growth after $24 \mathrm{hr}$. incubation at $37^{\circ} \mathrm{C}$. The results of FIC index were interpreted.

\subsection{Identification of NDM 1 gene existence}

DNA was extracted from bacterial isolates using bacteria DNA preparation kit supplied from Zymo Research, USA protocol. PCR reactions were performed in a final volume $30 \mu \mathrm{l}$ and PCR mixture composed of $15 \mu \mathrm{l}$ PCR master mix ready to use (Dream taq TM Green PCR. Master Mix (2X) 200rxn (Sigma, USA)), $1.5 \mu \mathrm{l}$ Forward primer, $1.5 \mu \mathrm{l}$ Reverse primer (were supplied from Zymo research, USA and $2 \mu$ l Nuclease free water. Reaction mixtures were mixed gently then centrifuged for $5 \mathrm{sec}$. and placed in a cold rack before adding $10 \mu \mathrm{l}$ of DNA extract. The thermal cycler (Gene Amp PCR 9700, Applied biosystem, USA) was programmed with the following cycling profiling according to Nordmann et al., $2011^{7} 10 \mathrm{~min}$ at $94^{\circ} \mathrm{C} ; 36$ cycles of amplification consisting of $30 \mathrm{~s}$ at $94^{\circ} \mathrm{C}, 40 \mathrm{~s}$ at $52^{\circ} \mathrm{C}$, and $50 \mathrm{~s}$ at $72^{\circ} \mathrm{C}$; and $5 \mathrm{~min}$ at $72^{\circ} \mathrm{C}$ for the final extension. DNA fragments were visualized after electrophoresis in $2 \%$ agarose gel. The isolates in which NDM-1 gene was not detected were further examined using the primers of Manchanda et al ${ }^{11}$ method and the thermal cycler (Gene Amp PCR 9700, Applied biosystem, USA) was programmed with the following cycling profiling as denaturation of DNA at $94^{\circ} \mathrm{C}$ for 3 min, followed by 30 cycles of denaturation, annealing and extension at $94^{\circ} \mathrm{C}, 60^{\circ} \mathrm{C}$ and $72^{\circ} \mathrm{C}$, respectively, for $30 \mathrm{~s}$ each. The final extension step was performed for 3 min at $72^{\circ} \mathrm{C}$. PCR products were electrophoresed in $1.5 \%$ agarose gel. Negative controls (NC) were added first before any of the samples are added to check for contamination in the master mix. Positive controls (PC) 
were added last after all samples are sealed to check for cross-contamination during sample preparation or addition. PCR products were then purified using Montage 96 well purification kit protocol for verification by DNA sequencing using the 3100 Applied Biosystems ${ }^{\circledR}$ gene analyzer and the resulting sequences were compared with those available on GenBank database (http://www.ncbi.nlm.nih.gov/BLAST).

\section{RESULTS}

CRAB Twenty seven clinical isolates (12 from males and 15 from females) that were selected in our study were of high frequent distribution among $\geq 60$ age group $(12 / 27)$ followed by the working age group (11/27). Clinical isolates were isolated as; (9) blood, (4) for each of urine, wound, sputum and endo-tracheal tube swab (ENT) and the last two were bronchial aspirate.

\subsection{Antimicrobial susceptibility of $A$. baumannii isolates}

Figure (1) shows the results of antibiotic susceptibility pattern of the twenty-seven CRAB clinical isolates.

Our research demonstrates complete resistance to all of amoxicillin+clavulanic acid, cefotaxime, ceftriaxone, cefipime and ceftazidime. High percentages of resistance were found to trimethoprim + sulphamethoxazole $26 \quad(96.30 \%)$ followed by gentamycin and azithromycin 25(92.59\%) for each and both of ciprofloxacin and levofloxacin 23(85.19\%). Moderate resistance was correlated to both of amikacin and tetracycline which was $21(77.78 \%)$. On the other hand, sensitivity was found towards both tigecycline and colistin as it was $16(59.26 \%)$ and $15(55.56 \%)$ respectively. No isolates were sensitive to all of antibiotics used. Results of MICs for the 27 CRAB were shown in table (1).
Figure (1): Results of antibiotic susceptibility pattern:

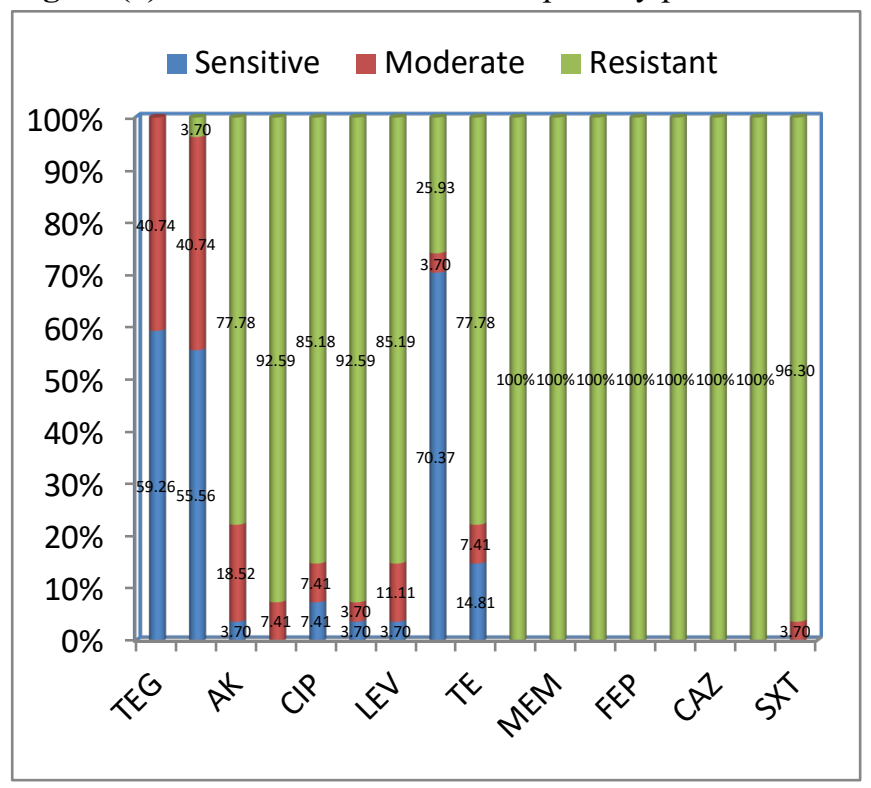

* Tigecycline (TIG), Colistin (COL), Amikacin (AK), Azithromycin (AZM), Ciprofloxacin (CIP), Gentamycin (CN), Levofloxacin (LEV), Nitrofurantoin (F), Tetracycline (TE), Imipenem (IPM) Meropenem (MEM), Amoxicillin-clavulanic acid (AMC) Cefepime (FEP), Cefotaxime (CTX). Ceftazidime (CAZ) and Ceftriaxone (CRO). and Tri-methoprim + sulphamethoxazole(SXT).

Table (1): the interpretation of MICs results by E-test method

\begin{tabular}{|l|l|l|l|l|l|l|}
\hline $\begin{array}{c}\text { Antibiotic } \\
\text { strip }\end{array}$ & \multicolumn{2}{|c|}{ Sensitive } & \multicolumn{2}{c|}{ Moderate } & \multicolumn{2}{c|}{ Resistant } \\
\hline & N & $\%$ & N & $\%$ & N & $\%$ \\
\hline TIG & 8 & 29.63 & 19 & $70.37 \%$ & 0 & 0 \\
\hline COL & 5 & 18.5 & 17 & $63 \%$ & 5 & 18.5 \\
\hline IPM & - & 0 & 16 & 59.26 & 11 & 40.74 \\
\hline MEM & - & 0 & 8 & 29.63 & 19 & 70.37 \\
\hline AK & - & 0 & 15 & 55.55 & 12 & 44.44 \\
\hline AMC & - & 0 & 0 & 0 & 27 & 100 \\
\hline FEP & 0 & 0 & 0 & 0 & 27 & 100 \\
\hline CTX & 0 & 0 & 1 & 3.7 & 26 & 96.3 \\
\hline CAZ & 0 & 0 & 3 & 11.11 & 24 & 88.89 \\
\hline CRO & 0 & 0 & 2 & 7.4 & 25 & 92.6 \\
\hline CN & 3 & 11.11 & 3 & 11.11 & 21 & 77.78 \\
\hline LEV & 4 & 14.80 & 9 & 33.33 & 14 & 51.86 \\
\hline SXT & 0 & - & 3 & 11.11 & 24 & 88.89 \\
\hline
\end{tabular}

* Tigecycline (TIG), Colistin (COL), Imipenem (IPM),

Meropenem (MEM), Amikacin (AK), Amoxicillin-clavulanic acid (AMC) Cefepime (FEP), Cefotaxime (CTX). Ceftazidime(CAZ) and Ceftriaxone (CRO), Gentamycin (CN), Levofloxacin (LEV) and Tri-methoprim + sulphamethoxazole (SXT).

- The break point value of each antibiotics was further compared with the criteria recommended by CLSI in $2015{ }^{12}$. 


\subsection{Combination of antibiotics}

The selected $27 \mathrm{CRAB}$ isolates were tested against eight different antibiotic combinations using the checkerboard method. Fractional Inhibitory Concentrations (FIC) values were calculated for each and interpretations of results were shown in table (2). It was resulted that all combinations show synergistic significant effect. The most effective combination which shows the highest synergism activity was (Colistin + Impipenem) $88.88 \%$ followed by (Colistin + Meropenem) $81.48 \%$ then (Tigecycline + Imipenem) $74 \%$. Similar additive effects were shown in all of (Tigecycline + Imipenem), (Tigecycline + Meropenem), (Tigecycline + Amikacin) and (Meropenem + Amikacin) 14.89\%. While, the highest indifferent effect was noticed in (Imipenem + Amikacin) antibiotic combination $25.93 \%$. None of the antibiotic combinations tested showed antagonistic activity. Table (2): FIC values calculated for the eight antibiotic combinations $(\mathbf{n}=\mathbf{2 7})$ *Interpretation of FIC results was as follows:- Synergism $\leq \mathbf{0 . 5}$, additive range $0.5-1$, Indifference or no interaction1 - 4 and Antagonism $>4 *$ Tigecycline (TIG), Imipenem (IPM), Meropenem (MEM), Amikacin (AK) and Colistin (COL).

Table (2): FIC values.

\subsection{Detection of NDM-1 gene existence}

The 27 CRAB Producing metallo- $\beta$-lactam isolates results for the detection of New Delhi Metallo- $\beta$ lactamase-1gene (NDM-1) existence after first PCR round according to Nordmann et al., $2011^{7}$ protocol showed 12 (44.44\%) positive cases. Positive PCR results showed a band at $621 \mathrm{bp}$ which refers to $N D M-1$ gene. The other negative 15 isolates underwent a second PCR round according to Manchanda et al ${ }^{11}$ protocol using different primer sequences.

The percentage of NDM-1 positive cases were 10/15 $(66.67 \%)$ with a band at $475 \mathrm{bp}$ which indicates the NDM-1 gene existence. Five isolates were still untypeable after using both protocols. Randomly chosen positive PCR products of both protocols were verified by DNA sequencing. The final consensus sequences were analyzed and submitted to NCBI GenBank (http://www.ncbi.nlm.nih.gov/BLAST) data base under the accession numbers, (HQ6526091), (MK682763.1), (MK682764.1), (MK682767.1), (MN251665.1), (MN251666.1) and (MN251670.1).

The alignments showed similarity ranged from $94 \%$ to $100 \%$ nucleotides identity. Further results were published in the previous research paper of Radwan SM et al., $2020^{9}$.

\begin{tabular}{|c|c|c|c|c|c|c|}
\hline \multicolumn{2}{|c|}{ Effect } & \multirow{2}{*}{$\begin{array}{c}\text { Synergism } \\
20\end{array}$} & \multirow{2}{*}{$\begin{array}{c}\text { Additive } \\
4\end{array}$} & \multirow{2}{*}{$\begin{array}{c}\text { Indifferent } \\
3\end{array}$} & \multicolumn{2}{|c|}{ Chi-Square } \\
\hline \multirow{2}{*}{ TIG+IPM } & No. & & & & \multirow{2}{*}{$\begin{array}{c}20.2 \\
22\end{array}$} & \multirow{2}{*}{$<0.001 *$} \\
\hline & $\%$ & $74.0 \%$ & $14.9 \%$ & $11.1 \%$ & & \\
\hline \multirow{2}{*}{ TIG+MEM } & No. & 19 & 4 & 4 & \multirow{2}{*}{$\begin{array}{c}16.6 \\
67\end{array}$} & \multirow{2}{*}{$<0.001 *$} \\
\hline & $\%$ & $70.4 \%$ & $14.9 \%$ & $14.9 \%$ & & \\
\hline \multirow{2}{*}{ TIG+AK } & No. & 18 & 4 & 5 & \multirow{2}{*}{$\begin{array}{c}13.5 \\
56\end{array}$} & \multirow{2}{*}{$0.001 *$} \\
\hline & $\%$ & $66.7 \%$ & $14.9 \%$ & $18.5 \%$ & & \\
\hline \multirow{2}{*}{ COL+IPM } & No. & 24 & 2 & 1 & \multirow{2}{*}{$\begin{array}{c}37.5 \\
56\end{array}$} & \multirow{2}{*}{$<0.001 *$} \\
\hline & $\%$ & $88.9 \%$ & $7.4 \%$ & $3.7 \%$ & & \\
\hline \multirow{2}{*}{ COL+MEM } & No. & 22 & 2 & 3 & \multirow{2}{*}{$\begin{array}{c}28.2 \\
22\end{array}$} & \multirow{2}{*}{$<0.001 *$} \\
\hline & $\%$ & $81.5 \%$ & $7.4 \%$ & $11.1 \%$ & & \\
\hline \multirow{2}{*}{$\mathrm{COL}+\mathrm{AK}$} & No. & 20 & 2 & 5 & \multirow{2}{*}{$\begin{array}{c}20.6 \\
67\end{array}$} & \multirow{2}{*}{$<0.001 *$} \\
\hline & $\%$ & $74.0 \%$ & $7.4 \%$ & $18.5 \%$ & & \\
\hline \multirow{2}{*}{ IPM+AK } & No. & 17 & 3 & 7 & \multirow{2}{*}{$\begin{array}{c}11.5 \\
56\end{array}$} & \multirow{2}{*}{$0.003 *$} \\
\hline & $\%$ & $63.0 \%$ & $11.1 \%$ & $25.9 \%$ & & \\
\hline \multirow{2}{*}{ МЕM+AK } & No. & 18 & 4 & 5 & \multirow{2}{*}{$\begin{array}{c}13.5 \\
56\end{array}$} & \multirow{2}{*}{0.001 * } \\
\hline & $\%$ & $66.7 \%$ & $14.9 \%$ & $18.5 \%$ & & \\
\hline
\end{tabular}




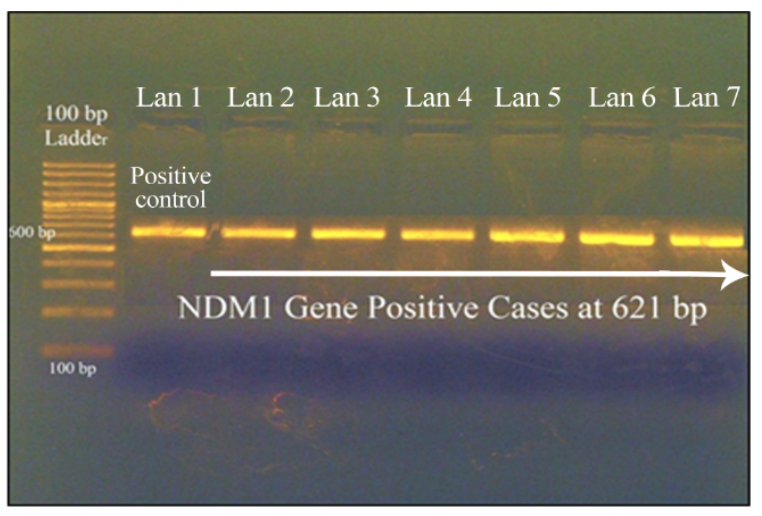

Figure (2): PCR amplification of NDM-1 gene according to Nordmann et al., 2011 ${ }^{7}$. The first lane (from the left) shows the DNA ladder. Lane 1 is the positive control. Lanes 2-7 are of identical patterns and show the same band at $621 \mathrm{bp}$ which refers to $N D M-1$ gene.

Table 3 shows high significant correlation between the sensitivity pattern of carbapenem resistant Acinetobacter baumannii isolates towards tigecycline and amikacin antibiotics and NDM1 gene existence identified by using Nordmann et al., $2011^{7}$ method.

\section{DISCUSSION}

Acinetobacter baumannii strains are resistant to various types of antibiotics used. The most common reasons for multi-drug resistance in Acinetobacter baumannii were the indiscriminate and inadequate use of antibiotics in both prophylaxis and minimally invasive surgery without drug sensitivity testing. Such reasons may have occurred either due to lack of advanced laboratory facilities, negligence on the part of general medical practitioners -who give treatment to most of infections- or patients of poor economic status and poor infection control practices. The present study reveals from its susceptibility testing that the resistance to antibiotics representing many classes e.g. (cell wall inhibitors, protein synthesis inhibitors and glycopeptides) increases; as all Acinetobacter baumannii isolates were resistant to most of tested antibiotics. This is in agreement with new researches conducted ${ }^{13-18}$. Current treatment options for CRAB are limited and suffer from pharmacokinetic limitations, such as high toxicity and low plasma levels [4]. These synergistic activities were similar to results conducted by Tuon et al., $2015^{5}$ and Isler et al., $2019^{4}$ in their researches for both (Colistin + Impipenem) and (Colistin + Meropenem). In contrast, Soudeiha et al., $2017^{19}$ results showed no synergistic effects but only

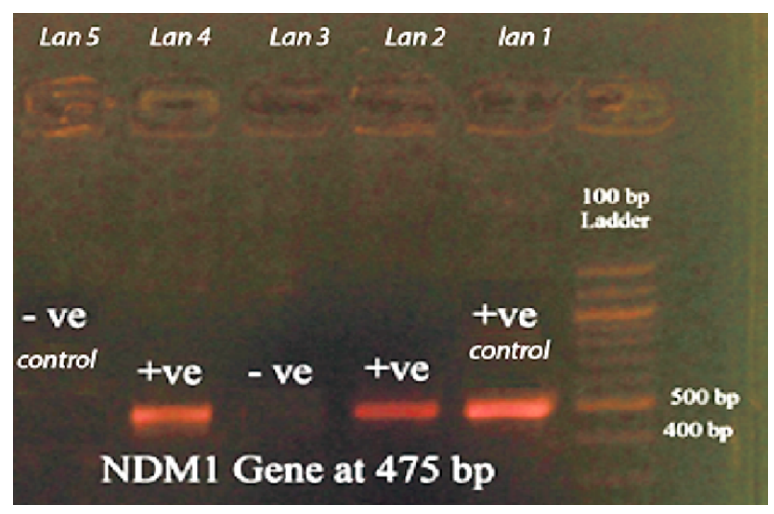

Figure (3): PCR amplification of NDM1 gene acc. to Manchanda et al., 2011 ${ }^{11}$. The first lane from the right shows DNA ladder. Lanes (1) shows positive control but lane (5) shows negative control. Lanes (2\&4) show similar patterns with the same band at $475 \mathrm{bp}$.

additive ones. In the present study, additive effects were shown in all of (Tigecycline + Imipenem), (Tigecycline + Meropenem), (Tigecycline + Amikacin) and (Meropenem + Amikacin). This is similar to what Isler et al., $2019^{4}$ documented in his study,

Colistin, Tigecycline, Minocycline and Amikacin) have been used for the treatment of CRAB infections. Moreover, Yadav et al., $2015^{20}$ documented that combination of high doses of imipenem in continuous infusion and aminoglycosides showed microbiological eradication.

various combinations of these drugs (Polymyxins, Two different methods were performed to detect the existence of New Delhi Metallo $\beta$ lactamase-1 gene (NDM-1). The first PCR procedure was performed according to Nordmann et al., $2011^{7}$ protocol. This protocol was also conducted in the studies applied by ${ }^{21,22}$. The negative isolates went through a second PCR round according to Manchanda et al., 2011 11 protocol ${ }^{23,24}$ also use the same also use the same primer sequence for NDM-1 identification. Our research resulted in high significant correlation between the sensitivity pattern of carbapenem resistant Acinetobacter baumannii isolates towards tigecycline and amikacin antibiotics and the existence of NDM-1 gene identified by using Nordmann et al.,2011 ${ }^{7}$ method. These results matched with what Isler et al., $2019^{4}$ documented that plasmid or chromosome carried NDM-1 gene, also carries a number of other genes conferring resistance to all aminoglycosides, macrolides and sulphamethoxazole. 
Table (3): Relation between NDM1 gene detected according to Nordmann et al., $2011^{7}$ method and the antibiogram of the carbapenem resistant Acinetobacter baumannii isolates.

\begin{tabular}{|c|c|c|c|c|c|c|c|c|c|}
\hline & & \multicolumn{6}{|c|}{ NDM1 typing } & \multirow{2}{*}{\multicolumn{2}{|c|}{ Chi-Square }} \\
\hline & & \multicolumn{2}{|c|}{ Negative } & \multicolumn{2}{|c|}{ Positive } & \multicolumn{2}{|c|}{ Total } & & \\
\hline & & $\mathbf{N}$ & $\%$ & $\mathbf{N}$ & $\%$ & $\mathbf{N}$ & $\%$ & $\mathbf{X}^{2}$ & P-value \\
\hline \multirow{2}{*}{ TEG } & Sensitive & 12 & 80.00 & 4 & 33.33 & 16 & 59.26 & \multirow{2}{*}{6.014} & \multirow{2}{*}{$0.014^{*}$} \\
\hline & Moderate & 3 & 20.00 & 8 & 66.67 & 11 & 40.74 & & \\
\hline \multirow{3}{*}{ COL } & Sensitive & 11 & 73.33 & 4 & 33.33 & 15 & 55.56 & \multirow{3}{*}{4.811} & \multirow{3}{*}{0.090} \\
\hline & Moderate & 4 & 26.67 & 7 & 58.33 & 11 & 40.74 & & \\
\hline & Resistant & 0 & 0.00 & 1 & 8.33 & 1 & 3.70 & & \\
\hline IPM & Resistant & 15 & 100.00 & 12 & 100.00 & 27 & 100.00 & $\mathrm{x}$ & $\mathrm{x}$ \\
\hline MEM & Resistant & 15 & 100.00 & 12 & 100.00 & 27 & 100.00 & $\mathrm{x}$ & $x$ \\
\hline \multirow{3}{*}{$\mathbf{A K}$} & Sensitive & 1 & 6.67 & 0 & 0.00 & 1 & 3.70 & \multirow{3}{*}{6.171} & \multirow{3}{*}{$0.046^{*}$} \\
\hline & Moderate & 5 & 33.33 & 0 & 0.00 & 5 & 18.52 & & \\
\hline & Resistant & 9 & 60.00 & 12 & 100.00 & 21 & 77.78 & & \\
\hline AMC & Resistant & 15 & 100.00 & 12 & 100.00 & 27 & 100.00 & $\mathrm{x}$ & $\mathrm{x}$ \\
\hline \multirow{2}{*}{$\mathbf{A Z M}$} & Moderate & 2 & 13.33 & 0 & 0.00 & 2 & 7.41 & \multirow{2}{*}{1.728} & \multirow{2}{*}{0.189} \\
\hline & Resistant & 13 & 86.67 & 12 & 100.00 & 25 & 92.59 & & \\
\hline FEP & Resistant & 15 & 100.00 & 12 & 100.00 & 27 & 100.00 & $\mathrm{x}$ & $\mathrm{x}$ \\
\hline CTX & Resistant & 15 & 100.00 & 12 & 100.00 & 27 & 100.00 & $\mathrm{x}$ & $\mathrm{x}$ \\
\hline $\mathrm{CAZ}$ & Resistant & 15 & 100.00 & 12 & 100.00 & 27 & 100.00 & $\mathrm{x}$ & $\mathrm{x}$ \\
\hline CRO & Resistant & 15 & 100.00 & 12 & 100.00 & 27 & 100.00 & $\mathrm{x}$ & $\mathrm{x}$ \\
\hline \multirow{3}{*}{ CIP } & Not done & 6 & 40.00 & 6 & 50.00 & 12 & 44.44 & \multirow{3}{*}{1.765} & \multirow{3}{*}{0.414} \\
\hline & Moderate & 2 & 13.33 & 0 & 0.00 & 2 & 7.41 & & \\
\hline & Resistant & 7 & 46.67 & 6 & 50.00 & 13 & 48.15 & & \\
\hline \multirow{3}{*}{$\mathrm{CN}$} & Sensitive & 1 & 6.67 & 0 & 0.00 & 1 & 3.70 & \multirow{3}{*}{1.728} & \multirow{3}{*}{0.421} \\
\hline & Moderate & 1 & 6.67 & 0 & 0.00 & 1 & 3.70 & & \\
\hline & Resistant & 13 & 86.67 & 12 & 100.00 & 25 & 92.59 & & \\
\hline \multirow{3}{*}{ LEV } & Sensitive & 1 & 6.67 & 0 & 0.00 & 1 & 3.70 & \multirow{3}{*}{3.757} & \multirow{3}{*}{0.153} \\
\hline & Moderate & 3 & 20.00 & 0 & 0.00 & 3 & 11.11 & & \\
\hline & Resistant & 11 & 73.33 & 12 & 100.00 & 23 & 85.19 & & \\
\hline & Not done & 10 & 66.67 & 9 & 75.00 & 19 & 70.37 & & \\
\hline $\mathbf{F}$ & Moderate & 1 & 6.67 & 0 & 0.00 & 1 & 3.70 & 0.873 & 0.646 \\
\hline & Resistant & 4 & 26.67 & 3 & 25.00 & 7 & 25.93 & & \\
\hline & Not done & 1 & 6.67 & 3 & 25.00 & 4 & 14.81 & & \\
\hline TE & Moderate & 2 & 13.33 & 0 & 0.00 & 2 & 7.41 & 3.134 & 0.209 \\
\hline & Resistant & 12 & 80.00 & 9 & 75.00 & 21 & 77.78 & & \\
\hline SYT & Moderate & 1 & 6.67 & 0 & 0.00 & 1 & 3.70 & 0831 & 360 \\
\hline $\mathbf{S X 1}$ & Resistant & 14 & 93.33 & 12 & 100.00 & 26 & 96.30 & 0.831 & 0.362 \\
\hline
\end{tabular}

* Tigecycline (TIG), Colistin (COL), Imipenem (IPM), Meropenem (MEM), Amikacin (AK), Amoxicillinclavulanic acid (AMC), Cefepime (FEP), Cefotaxime (CTX). Ceftazidime (CAZ), Ceftriaxone (CRO), Gentamycin $(\mathrm{CN})$, Levofloxacin (LEV), and Tri-methoprim + sulphamethoxazole (SXT). 


\section{CONCLUSION}

Acinetobacter baumannii is an opportunistic pathogen responsible for hospital-acquired infections. Marching on of MDR A. baumannii isolates is threatening the effectiveness of last resort antimicrobials. Each hospital should establish its own policy according to the resulted antibiogram, national and international guidelines of antibiotic usage. Amikacin, tigecycline, colistin and any other sensitive antibiotics should be used in a restricted way and cautiously to render them effective.

\section{Acknowledgement:}

We thank the three hospital laboratories for providing the isolates. We thank (MASRI) for providing the laboratories.

\section{Conflicts of interest}

The authors declare no conflict of interest.

\section{Ethical approval statement: NA}

\section{Author contribution}

SM and OM performed the experiments. SM, DA and SR wrote and revised the manuscript.

\section{Funding}

This research was not funded.

\section{REFERENCES}

1. Stewart S, Robertson C, Manoukian S, Haahr L, Mason H, McFarland A, Dacer S, Cook B, Graves N, Reilly J. How do we evaluate the cost of nosocomial infection/ The ECONI protocol: an incidence study with nested case-control evaluating cost and quality of life BMJ Open. 2019; 9:e0266687.

2. Elliott $\mathrm{C}$ and Justiz-Vaillant A. Nosocomial infections: A 360-degree review. Spring. 2018; 4(2): $72-81$.

3. Morris FC. Dexter C, Kostoulias X, Uddin MI, Peleg AY. The mechanisms of disease caused by Acinetobacter baumannii. Front. Microbial. 2019; 10(1601);1-20.

4. Isler B, Doi Y, Bonomo R, Paterson D. New treatment options against carbapenem-resistant Acinetobacter baumannii infections. Antimicrobial agents and chemotherapy. 2019 63(1): 1110-18.
5. Tuon F, Rocha J, and Merlini B. Combined therapy for multi-drug-resistant Acinetobacter baumannii infection - is there evidence outside the laboratory? Journal of Medical Microbiology. 2015; (64): 951-959.

6. Fomda BA, Khan A, Zahoor D. NDM-1 (New Delhi metallo $\beta$-lactamase-1) producing Gramnegative bacilli: Emergence \& clinical implications. 2014; 140(5): 672-678

7. Nordmann P, Poirel L, Carrer A, Toleman M, Walsh T. How to detect NDM-1 Producers. Journal of Clinical Microbiology. 2011; 49(2): 718-721.

8. Rathinasabapathi $\mathrm{P}$, Deepak SH, Rex A, Parani M. Molecualr detection of New Delhi metallo- $\beta$ lactamase-1 (NDM-1) Positive bacteria from environmental and drinking water samples by loop mediated isothermal amplification of bla NDM-1, Indian J Microbiol. 2015; 55(4): 400-405.

9. Radwan SM, Shabayek SM, El-Damacy DA and Hassanin OM. Microbiological Study on Nosocomial Acinetobacter baumannii Infection with Molecular Characterization of Metallo BetaLactamases, J Med Microb Diagn. 2020; 9(2) 1-6

10. Mulyaningsih, S., Sporer, F., Zimmermann, S., Reichling, J., \& Wink, M. Synergistic properties of the terpenoids aromadendrene and 1,8-cineole from the essential oil of Eucalyptus globulus against antibiotic-susceptible and antibioticresistant pathogens. Phytomedicine. 2010; 17(13), 1061-1066.

11. Manchanda V, Rai S, Gupta S, Rautela RS, Chopra R, Rawat DS, Verma N, Singh NP, Kaur IR, Bhalla P Development of TaqMan real-time polymerase chain reaction for the detection of the newly emerging form of carbapenem resistance gene in clinical isolates of Escherichia coli, Klebsiella pneumoniae, and Acinetobacter baumannii, Indian Journal of medical microbiology. 2011; 29 (3):249-253

12. CLSI Acinetobacter spp. M02 and M07. Clinical and Laboratory Standards Institute. January. 2015; 35 (3): 56-57.

13. Shamsizadeh $Z$, Nikaeen $M$, Esfahani $B N$, Mirhoseini SH, Hatamzadeh M, Hassanzadeh A. Detection of antibiotic resistant Acinetobacter baumannii in various hospital environments: potential sources for transmission of 
Acinetobacter infections. Environmental health and preventive medicine. 2017; 22(44): 1-7.

14. Blanco $\mathrm{N}$, Harris AD, Rock C, Johnson JK, Pineles L, Bonomo RA, Srinivasan A, Pettigrew MM, Thom KA, CDC Epicenters Program. Risk factors and outcomes associated with multidrugresistant Acinetobacter baumannii upon intensive care unit admission. Antimicrob Agents Chemother. 2018; 62 :01631-17

15. Lowe M, Ehlers MM, Ismail F, Peirano G, Piet J. Becker, Johann D. D. Pitout and Marleen M. Kock Acinetobacter baumannii Epidemiological and $\beta$ Lactamase data from two tertiary academic hospitals in Tshwane, South Africa. Front. Microbiol. 2018; 9:1280.

16. Haque M, Sartelli M, McKimm J, Abu Bakar M. Health care-associated infections - an overview. Infection and drug resistance. 2018; 11: 23212333

17. Almasaudi SB. Acinetobacter spp. As nosocomial pathogens: epidemiology and resistance features. Saudi Journal of Biological Sciences. 2018; 25:586-596.

18. Nasr P. Genetics, epidemiology and clinical manifestations of Multidrug-Resistant. Acinetobacter baumannii, Journal of Hospital Infection. 2019; 10 (1016): 1-24.

19. Soudeiha M Dahdouh E, Azar E, aSarkis D, Daoud Z. In vitro evaluation of the colistincarbapenem combination in clinical isolates of AA. Baumannii using the checkerboard, etest, and time-kill curve techniques. Front cell infect. microbiol. 2017; (7): 209.

20. Yadav R, Landersdorfer C, Nation R, Boyce J, Bulitta J. Novel approach to optimize synergistic carbapenem-aminoglycoside combinations against carbapenem-resistant Acinetobacter baumannii. Antimicrobial Agents and Chemotherapy. 2015; 59(4): 2286-2298.

21. Heydari F, Mammina C. and Koksal F. NDM-1producing Acinetobacter baumannii ST85 now in Turkey, including one isolate from a Syrian refugee, Journal of Medical Microbiology. 2015; 64: 1027-1029.

22. Abidin NZ, Sulong A, Alfizah H, Muttaqillah N, Ding $\mathrm{CH}$. Molecualr detection of the New Delhi metallo- $\beta$-lactamase- 1
Enterobacteriaceae isolates in a tertiary medical center. Malaysian J Pathol. 2015; 37(3): 227-232.

23. Yong D, Toleman MA, Giske CG, Cho HS, Sundman K, Lee K, et alCharacterization of a new metallo- $\beta$-lactamase gene, bla $N D M-1$, and a novel erythromycin esterase gene carried on a unique genetic structure in Klebsiella pneumonia sequence type 14 from India. Antimicrob. Agents Chemother. 2009; 53: 5046-5054.

24. Chandar B, Poovitha S, Ilango K, Mohankumar R, Parani M. Inhibition of New Delhi metallo- $\beta$ lactamase 1 (NDM-1) producing escherichia coli IR-6 by selected plant extracts and their synergistic actions with antibiotics. Front Microbiol. 2017; (8): 1580. 